\title{
Simulating Mode Transitions during Breakdown in Liquids
}

\author{
Brian T. Murphy, Robert E. Hebner \\ The University of Texas at Austin \\ Center for Electromechanics \\ 1 University Station \#R7000 \\ Austin, TX 78712, USA \\ and Edward F. Kelley \\ KELTEK, LLC \\ PO Box 866 \\ Niwot, CO 80544, USA
}

\begin{abstract}
The primary new information from this investigation is the simulation of a transition from one streamer mode to another during propagation. The transition is characteristic of experimental data, but has not heretofore been described in a model. This demonstrates that the conceptual framework describing prebreakdown streamers in liquids as stochastic growth of a branching fractal tree in point-plane geometry is capable of simulating a wide range of streamer propagation behaviors in insulating liquids. The work also improved the approach in the model for scaling the potential at nearby grid points and explored some of the influences of computational choices, specifically needle length and grid size, on the predicted results.
\end{abstract}

Index Terms - Liquid breakdown, mode transition, modeling, streamers.

\section{INTRODUCTION}

THIS paper is an extension of the description of prebreakdown streamers in liquids as stochastic growth of a branching fractal tree in point-plane geometry [1-3]. The first investigation [1] developed the approach and demonstrated the predicted morphology of a streamer initiating from a point anode matched experimental observations. In that study, however, growth was assumed to a have a dependence on the electric field, $E$, of the form $E^{p}, p=1,2,3$, or 4 , with the higher powers apparently yielding better correlation. This work was seminal because it represented observed behavior quite well. It did, however, raise questions as to why it worked as well as it did with an unphysical assumption about the dependence on the electric field. No experiments had suggested power law dependence.

An obvious refinement [2] was to simulate the experimentally observed results with a more physical set of growth assumptions. This refinement maintained the assumption from [1] that the field outside of the streamer can be calculated assuming the streamer is an electrical conductor, as this assumption was validated by experiment [4]. With this assumption, only a linear dependence on the field strength was assumed. The second key assumption was that a streamer growth step occurred when an electron existed in a location such that the energy gained in a free path was sufficient to produce ionization upon impact with a molecule in the liquid. This assumes a dynamic, non- equilibrium situation [5], as would be expected during the breakdown processes.

This work expands on the previous investigations by showing that the model can also predict an important attribute of streamer growth that was not predicted in the earlier investigations. From the experimental data, it is clear that the streamer transitions from one mode of growth to the other during propagation from one electrode to another [6]. The modes studied in this work are the second and third cathode modes, which differ in morphology. The second mode is bush-like, while the third has a typical streamer shape. In addition, the third mode propagates about an order of magnitude faster than the second. This investigation shows that transition can be simulated by enhancing the probability of stepping to the high field areas as the streamer grows. This suggests that the previous work may have suppressed transition by the propagation assumptions that were used.

\section{SUMMARY OF COMPUTATIONAL APPROACH}

Figure 1 illustrates the volume employed for the calculation. The volume is discretized on a rectangular grid. For example, a cubical volume may be modeled using a $128 \times 128 \times 128$ grid in the $x, y$, and $z$ directions, respectively. This would result in just over two million uniformly spaced grid points. In Figure 1, the top of the box is considered to be a planar electrode having a uniform potential of 0 . A needle, also at zero potential, extends down from the top of 
the box. The electrode is the bottom of the box, and is assigned a uniform potential of 1 . The distribution of electric potential throughout the box is determined numerically by solving Laplace's equation in three dimensions,

$$
\nabla^{2} \phi=0 \text {. }
$$

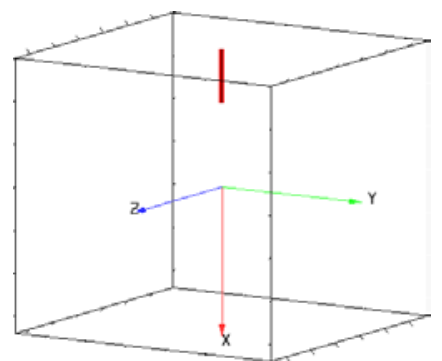

Figure 1. Sample volume used for the streamer simulation. The top of the box is an anode plate. The bottom of box is a counter electrode plate. The heavy red line is a needle at the same potential as the top plate.
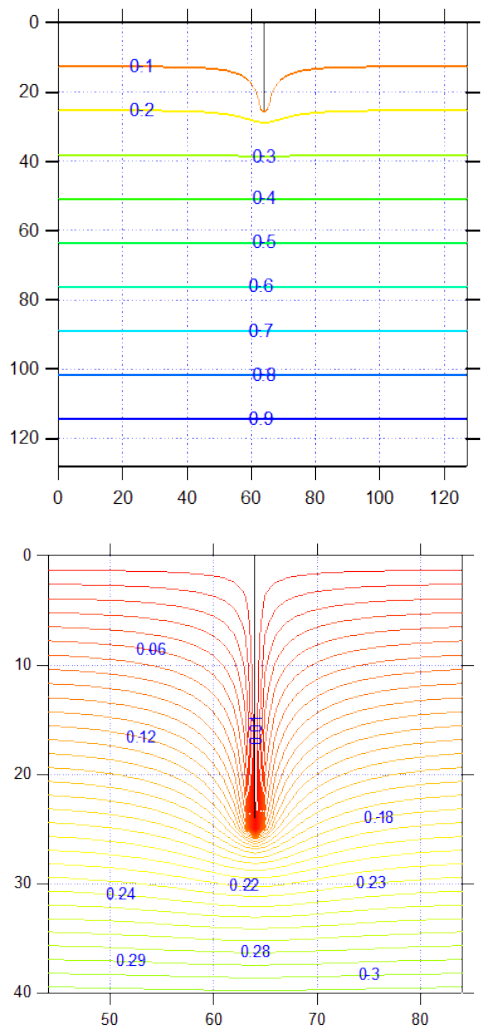

Figure 2. Top: Computed potential in the plane of the needle on a $128^{3}$ mesh, prior to streamer growth. The needle length is 25 points. Bottom: A more detailed enlargement of the region around the needle. Axis values are the grid points used in the calculations.

The solution of equation (1) is a spatial linear algebra problem of $m$ equations in $m$ unknowns, where $m$ is the number of grid points at which the potential is not predefined. When $\mathrm{m}^{3}$ is large, the solution can be efficiently determined using a Gauss-Seidel iterative solution algorithm. Since the linear equations being solved are rather simple and repetitive, the equation coefficient matrix need not actually be stored in its entirety in the computer implementation. Equation coefficients are instead generated when needed. Determining a solution to equation (1) then requires only multiplying the coefficient matrix times an assumed solution vector a suitable number of times to achieve convergence. Figure 2 illustrates the solution to equation (1) performed on a mesh of size $128^{3}$ with a needle having a length of 25 points in the $x$ direction. The potential distribution of Figure 2 also reveals that a periodic boundary condition has been employed at the sides of the box. This is as if the box had an identical neighboring box joined to it at each side wall. Envision an infinite grid of boxes extending in two dimensions, each with its own needle. Done this way, the model does a good job of simulating two parallel infinite plates as long as the box size is big enough to provide adequate distance between needles.

A significant aspect of the computed potential is its distribution right below the tip of the needle. This is where the streamer is known to initiate. Figure 3 examines the potential along a straight line from the needle's tip to the counter anode. Along this line, $\phi$ starts at zero and quickly trends to values having a constant gradient of one. This is the expected result $[7,8]$ with a small perturbation in what are elsewhere parallel plates, i.e., the field is uniform suitably far from the perturbation.
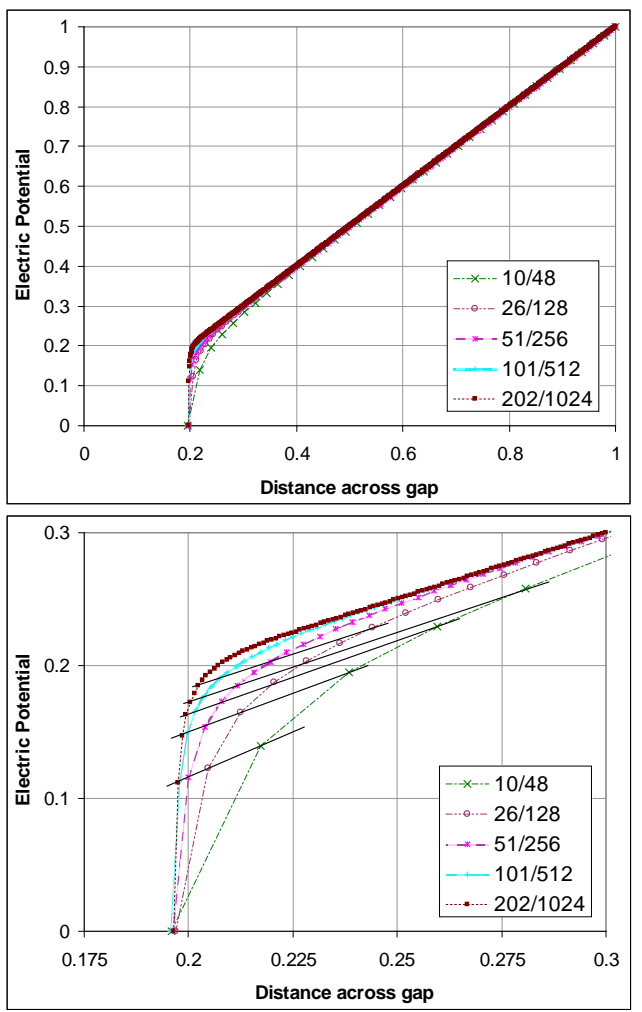

10/48 means needle tip is 10 points from the anode plate on a $48^{3}$ mesh

Figure 3. Electric potential along a line from needle tip to counter anode for a range of mesh densities. Needle tip is at a common point 0.196 across gap.

Figure 3 shows the variation of $\phi$ for a range of mesh densities. The coarsest, $48^{3}$, required about $15 \mathrm{~s}$ of CPU time to compute. The densest, $1024^{3}$, required $120,000 \mathrm{~s}$ of total CPU time (about one hour to run using $32 \mathrm{CPU}$ 's). This particular problem has been found to attain nearly ideal efficiency gains in a distributed memory parallel computing environment (FORTRAN, in conjunction with 
an MPI parallel library [9]). For this application, a box-like volume like that in Figure 1 is conveniently partitioned into a series of rectangular bricks, or sub-volumes. An MPIenabled computer program runs simultaneously on each of a set of CPUs. A single CPU operates on each brick of the volume. The memory, which is "distributed" in this case, is the solution vector for the potential $\phi$. Each CPU needs $\phi$ only within its corresponding sub-volume (note, there is a small amount of overlap in the $\phi$ requirement at sub-volume boundaries, and this is managed by the MPI library).

The effect that increasing mesh density has on $\phi$ is interesting. While moving away from the tip of the needle, the gradient quickly approaches 1 , as expected. The transition happens faster with a denser mesh. In each calculation case, the needle is the same absolute length, and the needle itself is a single line of mesh points. So for this case study, the needle is effectively thinner with a denser mesh.

At distances well removed from the needle, Figure 2 shows that the "free-field" potential at any given point is equal to the distance across the gap. This is a result of the gradient being uniform absent the needle. When the curves in Figure 3 are re-plotted as a fraction of the free-field potential versus mesh point number (Figure 4), nearly the same value is found regardless of mesh density. This means the potential attains roughly the same fraction of its freefield value in the same number of mesh points regardless of mesh density. For all cases, it takes about 10 mesh points to transition to the free field level. In conjunction with this, note also in Figure 3 (where points are plotted versus distance) that the points of a common mesh point number can be connected with a straight line. In addition, these lines appear to be directed at a common point of $(1,1)$ at the counter electrode.

Figure 3 also shows that, as mesh density increases, the value of $\phi$ at the first mesh point below the needle is converging to a value of about 0.12 (or about $55 \%$ of the free-field potential per Figure 4 ). This implies that as one approaches the tip of an infinitely thin needle, the gradient increases to infinity (i.e., 0.12/0), as expected.

By generating additional figures similar to Figure 4 for other needle lengths, it was possible to devise the following approximate expression to compute the value of $\phi$ at the first grid point below the tip of the needle. This expression will be used in later sections of this paper. It was found to be accurate within a few percent for all cases studied.

$$
\phi_{n 1} \approx 0.572 \frac{n+1}{N-3} \text {. }
$$

where

$n=$ value of $x$ at the needle tip

$N=$ mesh size in the $x$ direction

At two grid points below the needle the following expression applies:

$$
\phi_{n 2} \approx 0.737 \frac{n+2}{N-3} \text {. }
$$

Equation (2) can be used to approximate the potential at the nearest free point below the tip of a growing streamer. It is thus evident that as the streamer lengthens, the potential one point in front of the streamer tip is increasing. The streamer may then be attracted more strongly as it approaches the counter electrode. During simulation, how this influences streamer growth also depends on the details of the growth model. Figure 5 illustrates the linear nature of the increase in potential just ahead of an advancing streamer for progressively less bushy streamers. This behavior is a consequence of the electro-optical measurements that show that the streamer can be modeled as an electrical conductor [10].

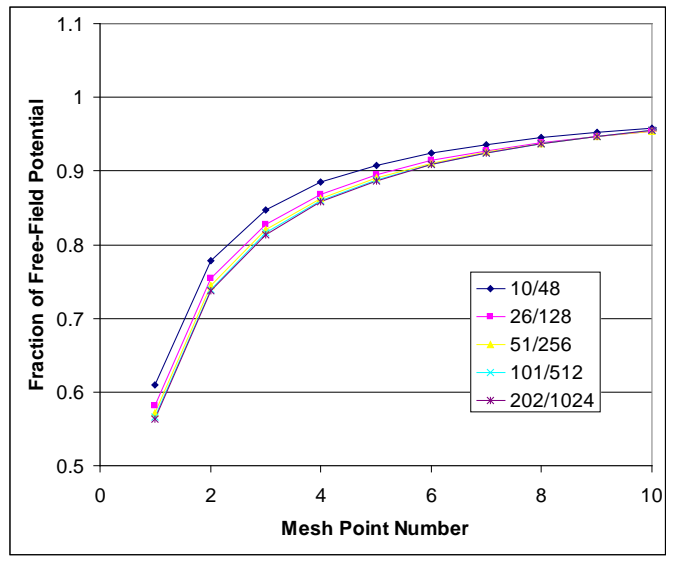

Figure 4. Gradient of electric potential versus mesh point number.

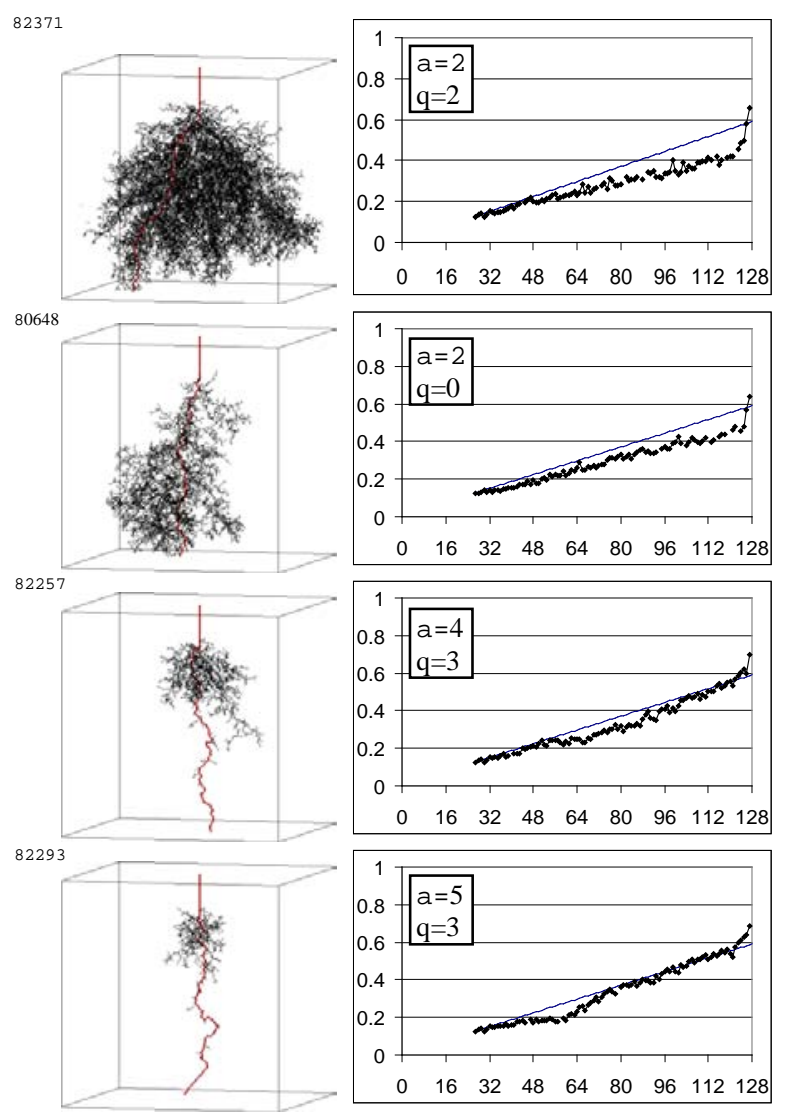

For each case $\phi_{c}=.06$ and needle length $n=26 ; a$ and $q$ are defined in equation (4).

Figure 5. Samples of potential at the point one grid step in front of the advancing streamer. In the 2-D graphs, the abscissa is the vertical $x$ coordinate, the ordinate is electric potential. Solid lines are of equation (2). 


\section{MODEL FOR STREAMER GROWTH}

As stated earlier, the potential of the conducting needle is 0 (and 0 also on the top electrode). Calculation of the $\phi$ distribution provides the value of $\phi$ at all free points throughout the volume. It is assumed that streamer growth will be from a point on the needle to a free point "adjacent" to the needle. Here "adjacent" means points which are one grid step from the needle. It is also assumed that for growth to an adjacent point to be possible, the value of $\phi$ at the adjacent point must exceed a certain value designated the cutoff value, $\phi_{c}$. This is equivalent to requiring that the voltage gradient must exceed a minimum threshold for growth to be possible. This assumption appears consistent with what has been observed in laboratory experiments [11].

In the general case, there will be multiple adjacent points where $\phi>\phi_{c}$. Streamer growth is treated as a purely stochastic process. This means that among the set of adjacent points at which $\phi>\phi_{c}$, points will be randomly selected to be growth points. The details of the random point selection process are described in the next section. The definition of the needle is then extended to include all growth points (i.e., the streamer). At each of the newly added growth points, the potential $\phi$ is set to 0 , and the $\phi$ distribution for all remaining free points is recomputed. So the model for streamer growth is essentially as follows:

1. Compute the distribution of $\phi$.

2. Randomly add some growth points to the streamer.

3. Recalculate the distribution of $\phi$.

4. Repeat until the gap is bridged.

As long as an appropriate point selection method is used, the prevailing gradient of the potential should cause the streamer to grow generally towards the opposite electrode. The simulation is halted when the streamer bridges the gap. At this point, much higher current flows and a different model is required [12].

\section{POINT IDENTIFICATION AND LENGTH CORRECTION}

Before discussing the random point selection process, the method of identifying "adjacent” points will be described. This is necessary because calculations show that the selection process influences the characteristics of streamer growth. There are 27 grid points shown in Figure 6. The point in the center, which is un-numbered, is considered to be part of the streamer. It is seen that 26 points surround the streamer point, which are all one grid step from the streamer point. Each of these points is a possible growth point. Each is assigned an identification number 1 through 26. Some of these points may already be on the streamer. Indeed, at least one must be in order for the streamer to reach the center point. As an example, consider the streamer to be coming straight down, passing through point 1 (and also the point directly above point 1 ) to reach the center point. A free point located at the point labeled 6 could be rightfully tagged with an identification number 17 , 6 , or 18. This ambiguity is dealt with by working in an outside-in and top-down fashion. All free points which can be identified as being corner types (19-26) are tagged the corresponding value. Then all free points which can be identified as being in the middle of an edge $(7,8,15,17$, $11,12,13,14,9,10,16,18)$ are tagged as such. This second tagging step may replace some tags assigned in the first step. As a third step, point types (3-6) are tagged. Then finally points of types $(1,2)$ are tagged as such.

Points (3-6) are each exactly 1.0 grid steps from the streamer. Points (11-14) are a distance of $\sqrt{2}$ grid steps from the streamer. Since they are farther from the streamer, $\phi$ will tend to be greater in value than at points (3-6). The value of $\phi$ at point 11 , for example, is divided by 1.332 to approximate the value it would have if it were actually one step from the streamer instead of $\sqrt{2}$ steps. The value of 1.332 is obtained by passing a second order polynomial through the points from equations (2) and (3), and evaluating this curve at a grid distance of 1.414 units. In a similar fashion, points (23-26) are $\sqrt{3}$ grid steps from the streamer, and $\phi$ at these points is divided by 1.555 . Correcting for distance in this fashion is analogous to evaluating the gradient of the potential toward each point. This adjustment is likely more physical than the approach employed in [1], where they divided by the distance (either $\sqrt{2}$ or $\sqrt{3}$ ), but the differences in growth dynamics are relatively small.

It is desired to preclude the possibility of simultaneous streamer growth to the same free point from multiple points on the streamer. Therefore, throughout the entire volume all points are assigned a fixed color, red or black, in the alternating sense of a chessboard. For each step of streamer growth, only the red or black set of points is considered for growth destinations (which set is selected randomly).

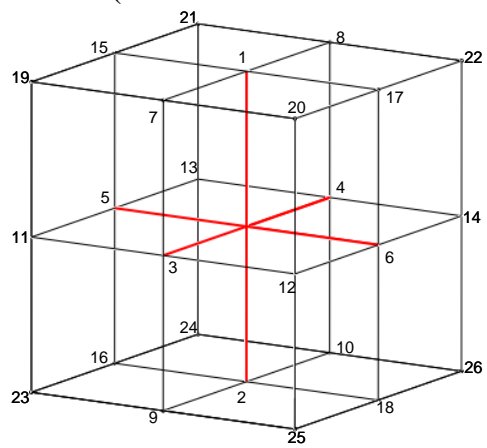

Figure 6. Identification scheme for adjacent points.

\section{GROWTH ASSUMPTIONS}

It was found [3] that a wide variety of streamer forms could be produced by adjusting the potential cutoff $\phi_{c}$ and a cutoff $r n_{c}$. To "randomly" select say, $10 \%$ of the available points, each is assigned an $r n$ (i.e., random number) from 0 to 1 , and points having $r n<0.1$ become growth points [3]. This could be termed a two-parameter growth model ( $\phi_{c}$ and $r n_{c}$ ). Figure 7 shows a sample taken from Figure 3 of [3] and a reproduction calculated as part of this work. For the same parameters, our analysis produces streamers which branch out a bit more and are bushier. This minor 
difference is primarily a result of the different length adjustment.

In general, a two-parameter model is obtained by exponentiating $\phi$ to a power greater or less than 1 before comparison to the rn's. The power of exponentiation becomes a second parameter. This is the approach used in this investigation.
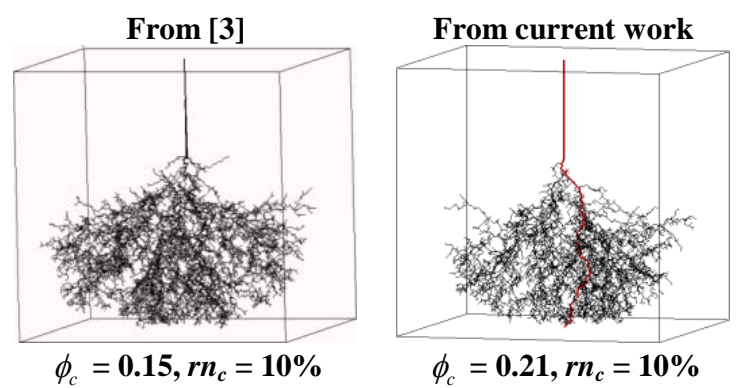

Figure 7. Comparison of streamer from [3] with current work.

As such, points with larger $\phi$ values have a higher probability of being selected as growth sites. This tends to result in streamers which are less bushy and directed toward the counter anode plate. Figure 5 shows that, overall, $\phi$ values at adjacent points start out small and grow larger as the streamer grows, eventually attaining a value of about 0.6 when reaching the counter anode.

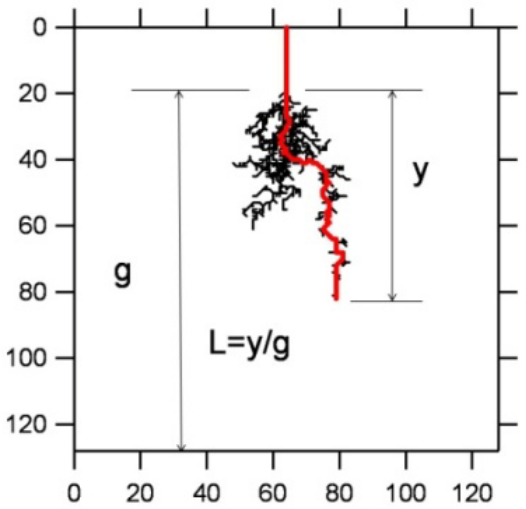

Figure 8. Definition of length of a growing streamer. The numerical values for the horizontal and vertical axes are the grid values used in the calculation.

There are convenient statistical aspects of using $r n$ 's in the range $\{0: 1\}$ having a uniform distribution, combined with $\phi$ values also in the range $\{0: 1\}$. First, the probability that any $\phi$ value will exceed its corresponding $r n$ is equal to $\phi$. Second, for any given population of $\phi$ values, the sum of the $\phi$ values is the average number of $\phi$ values which will exceed their corresponding $r n$ (i.e., be selected as growth sites). For example, if the $\Sigma \phi=3$, then on average about three points will be selected as growth sites. If $\Sigma \phi=0.5$, then roughly half the time no points would be selected. When no points are selected, a new set of $r n$ 's is generated for comparison, and this is repeated until at least one growth site is selected. Once one or more growth sites are selected, these points are added to the streamer, and the potential distribution in the volume is recalculated in preparation for the next step of growth.
Raising the $\phi$ values to a power greater than 1 will make them smaller, and a power less than 1 makes them larger. In either case, they will remain in the range $\{0: 1\}$. Negative powers should not be used since this will result in values greater than 1. Exponentiation in this fashion, where $\phi$ is replaced with $\phi^{p}$, changes the distribution of the values (i.e., both the mean and variance are altered).
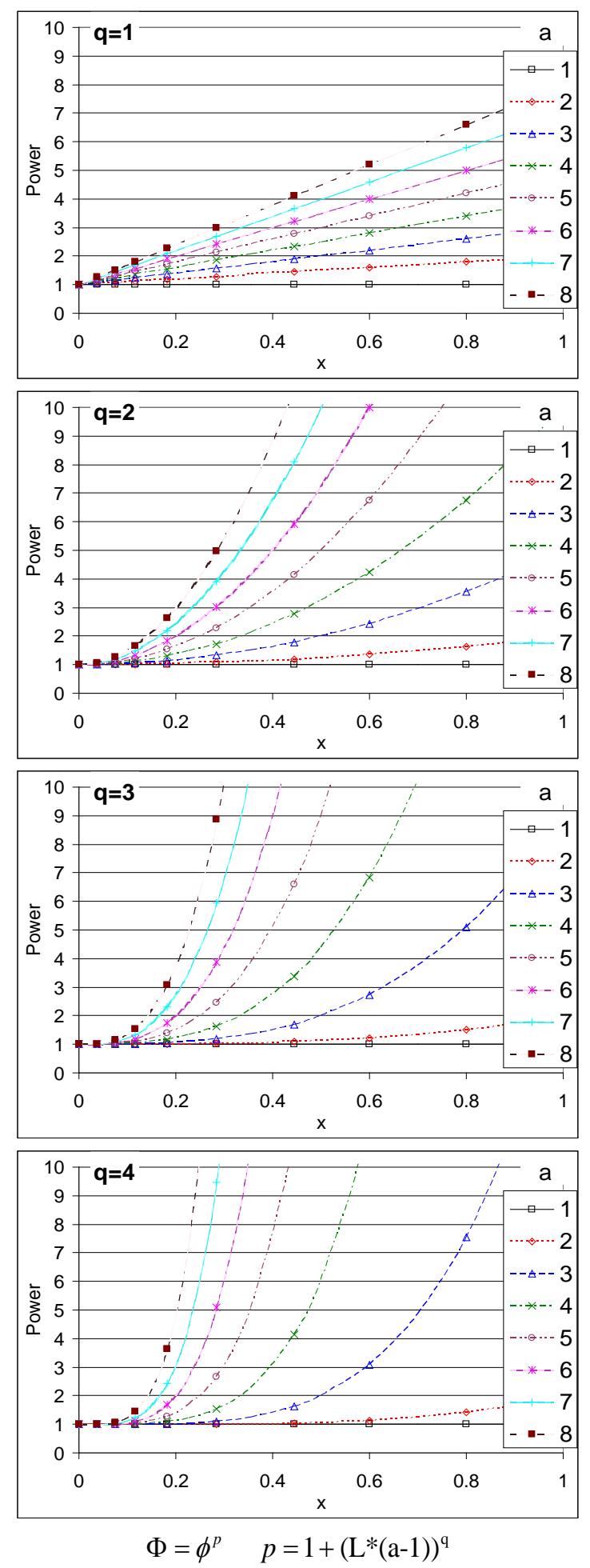

Figure 9. Exponentiation power as a function of streamer length. 
It was demonstrated in [2], that the computational effect of changing the value of the exponent on $\phi$ is the same as making more physical assumptions concerning the random selection process. Consequently, it is reasonable to use this approach for computational efficiency.

Changing the distribution of the $\phi$ values affects the relative likelihood of small and large values being selected for growth. As will be evident in the next section, when point selection is biased toward larger $\phi$ values, streamer growth is less dense.

As a final parameter of our streamer growth model, we employ a method similar to one examined in [3]. We have the power of exponentiation be a function of streamer length. Figure 8 and Figure 9 illustrate how the power varies as a function of length. This makes the method a three-parameter model,

$$
\Phi=\phi^{p}, p=1+[L \cdot(a-1)]^{q} .
$$

\section{STREAMER GROWTH CHARACTERISTICS}

For the presently devised basic calculation procedure, there are two analysis parameters which may influence streamer growth: the cutoff, $\phi_{c}$, which is the minimum required $\phi$ value for streamer growth, and the power of exponentiation, $p$, applied to the $\phi$ values prior to comparison to random numbers.

For all calculation cases, there are two important values of electric potential that exist at the start of the simulation:

1. The value of $\phi$ one grid point below the top anode plate, but well removed from the needle. This is referred to as $\phi_{1}$ and is equal to $1 /(N-1)$ where $N$ is the mesh size.

2. The value of $\phi$ one grid point below the tip of the needle. This is referred to as $\phi_{n}$ and can be approximated using equation (2).

Figures 10a-10d show an example of the effect of $\phi_{c}$ on streamer growth. In this example, $\phi_{1}=1 /(\mathrm{N}-1)=0.02$, so with $\phi_{c}$ less than this as in plot (a), stray streamers are seen to originate from the top anode plate and along the length of the needle. If $\phi_{c}$ were greater than $\phi_{n}$, the streamer would not grow at all since $\phi_{n}$ is the maximum $\phi$ value adjacent to the needle. Here the value of $\phi_{n}$ is 0.13954 for the $48^{3}$ cases in Figure 10, as compared to 0.1398 from equation (2). Also shown are cases with a $128^{3}$ sized mesh. These results show that streamer growth is only mildly sensitive to the value of $\phi_{c}$.

In Figures $10 \mathrm{~b}$ and $10 \mathrm{~g}$, evidence of the periodic boundary condition employed at the side walls can be seen. When the streamer reaches a side wall and leaves the box, the streamer enters the box on the opposing wall (i.e., wraparound).

Figure 11 shows a series of calculations examining the effect of needle length on streamer growth. As was the case for $\phi_{c}$, streamer growth does not appear to be sensitive to the length of the needle. This agrees with the expected result, as the field in the unperturbed liquid between the streamer and the electrode toward which it is propagating is strongly influenced by the shape of the streamer and much less influenced by the initial electrode geometry.

$48^{3}$ mesh, needle tip at $x=10, p=2$ a) $\phi_{c}=\mathbf{0 . 0 1 5}$

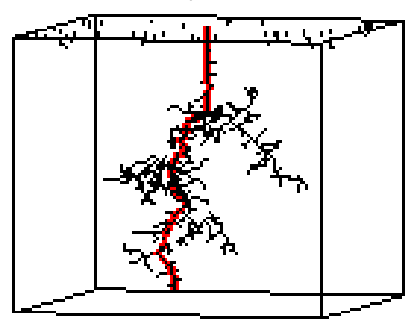

c) $\phi_{c}=\mathbf{0 . 0 9}$

$128^{3}$ mesh, needle tip at $x=26, p=2$

e) $\phi_{c}=\mathbf{0 . 0 0 9}$

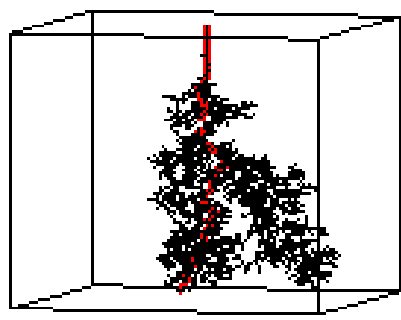

g) $\phi_{c}=\mathbf{0 . 0 9}$

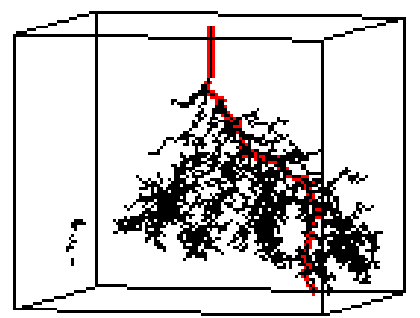

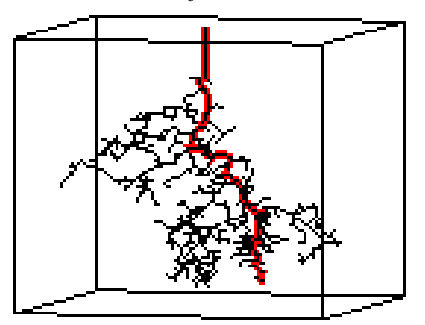

b) $\phi_{c}=\mathbf{0 . 0 3}$

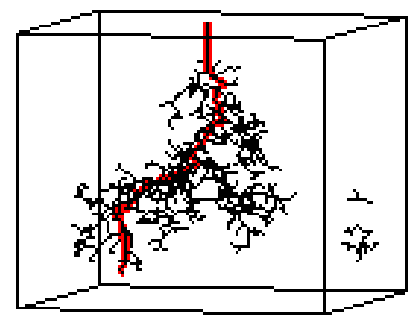

d) $\phi_{c}=\mathbf{0 . 1 3 7 5}$

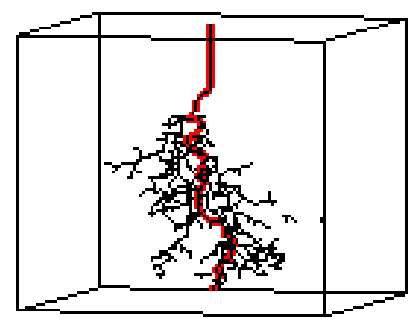

f) $\phi_{c}=\mathbf{0 . 0 6}$

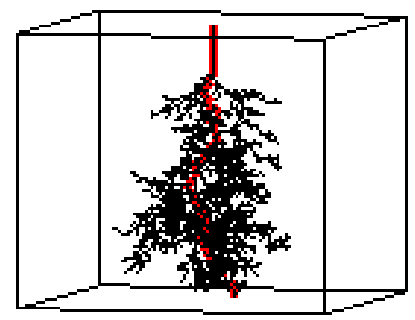

h) $\phi_{c}=\mathbf{0 . 1 2}$

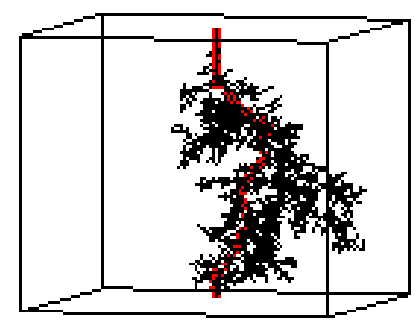

Figure 10. Effect of $\phi_{c}$ on streamer growth.

Figure 12 examines the effect of mesh size on streamer growth. In these cases, the length of the needle as a fraction of the mesh size was maintained constant. So the needle looks the same length in each image. Equation (2) indicates that the value of $\phi_{n}$ will be about the same for each case. The value of $\phi_{c}$ was chosen to be $50 \%$ of $\phi_{n}$. Mesh size does not have a strong effect on the appearance of the streamer. Even though the $N=512$ image appears denser, the streamer is actually less dense as it contains fewer points as a percentage of the volume's points. One thing which is strongly affected is the execution time, which increases exponentially with mesh size. Nearly all computation effort is spent re-solving Laplace's equation after each step of growth. This is done in parallel on multiple processors. 
$128^{3}$ mesh, $p=2, \phi_{c}=\phi_{1} / 2, n=x$ at needle tip
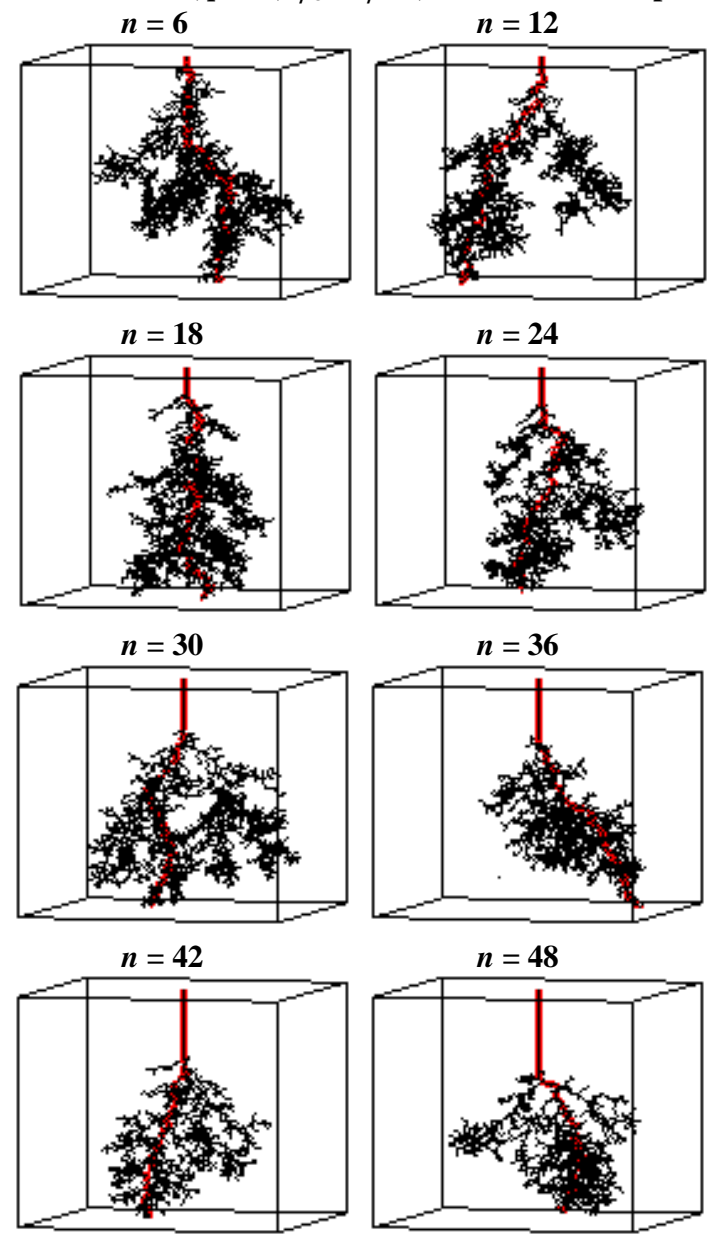

Figure 11. Illustration of effect of needle length on streamer growth.

$\boldsymbol{p}=2, \phi_{c}=\phi_{n} / 2$

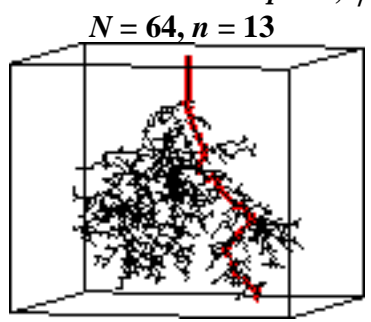

$N=256, n=52$
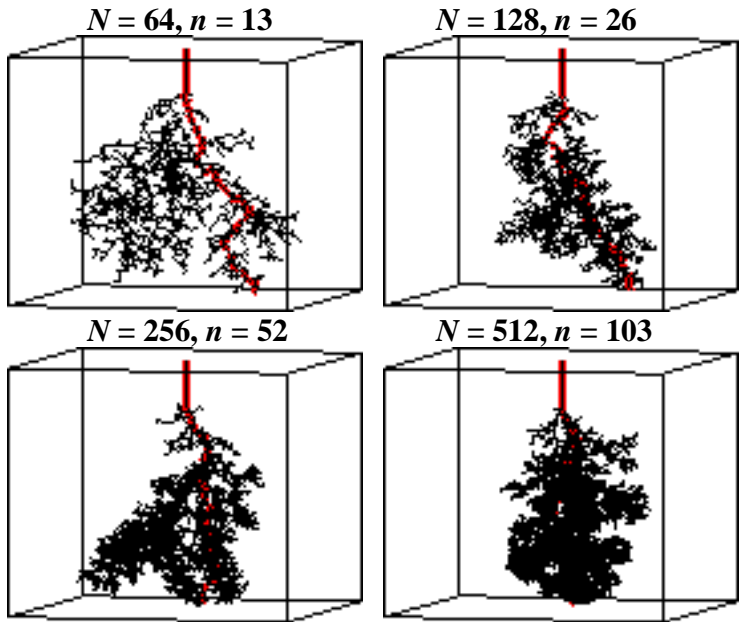

$N=512, n=103$

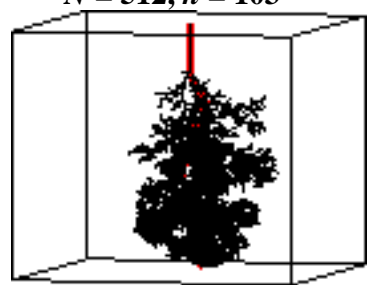

\begin{tabular}{|c|c|c|c|r|}
\hline Mesh Size & $\begin{array}{c}\text { Number of } \\
\text { Growth } \\
\text { Steps }\end{array}$ & $\begin{array}{c}\text { Number of } \\
\text { Streamer } \\
\text { Points }\end{array}$ & $\begin{array}{c}\% \text { of Total } \\
\text { Points }\end{array}$ & $\begin{array}{c}\text { Execution } \\
\text { Time }\end{array}$ \\
\hline 64 & 221 & 1908 & $0.73 \%$ & $26 \mathrm{~min}$ \\
\hline 128 & 421 & 5110 & $0.24 \%$ & $84 \mathrm{~min}$ \\
\hline 256 & 911 & 23588 & $0.14 \%$ & $1,210 \mathrm{~min}$ \\
\hline 512 & 2435 & 108416 & $0.08 \%$ & $29,149 \mathrm{~min}$ \\
\hline
\end{tabular}

Figure 12. Illustration of effect of mesh size on streamer growth.
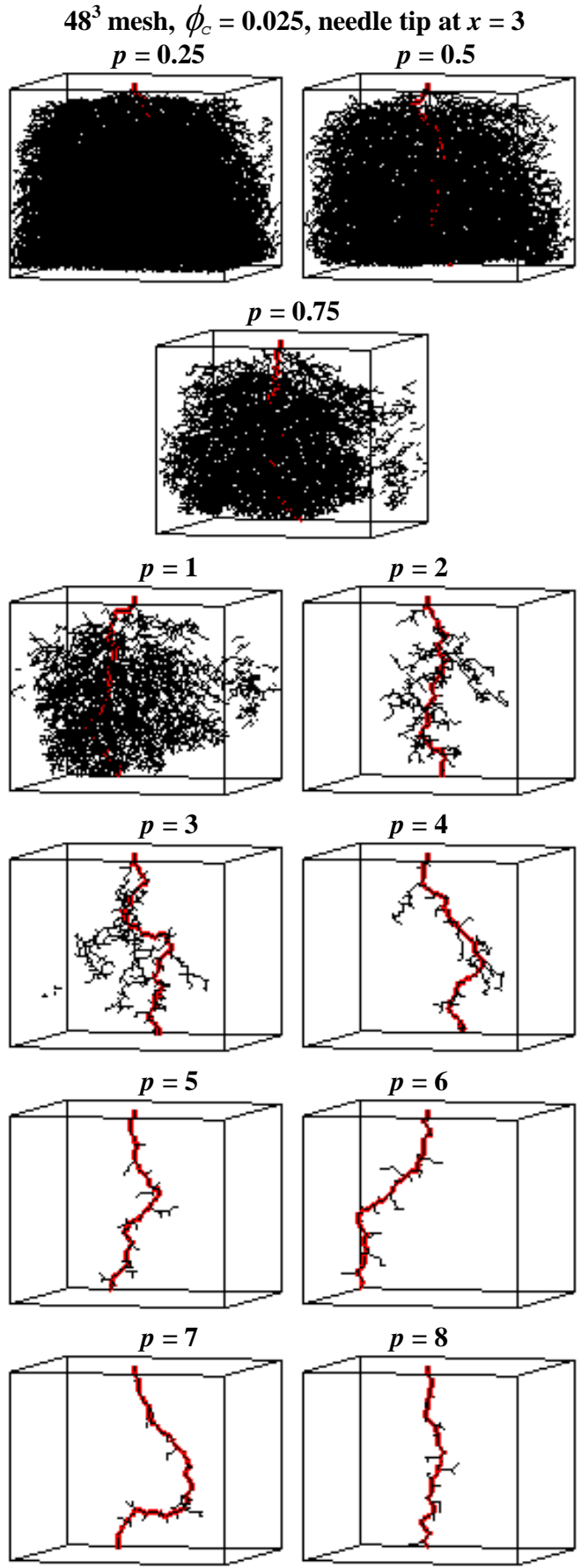

Figure 13. Cases with a short needle for a range of exponential powers.

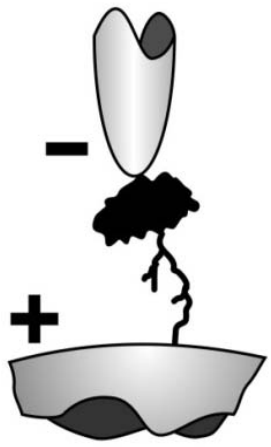

Figure 14. Illustration of $4^{\text {th }}$ cathode mode (Hebner [6]). 
Mesh $=128 \times 256^{2}, \phi_{c}=\phi_{n} / 2$, needle tip at $x=26$

$q=2$

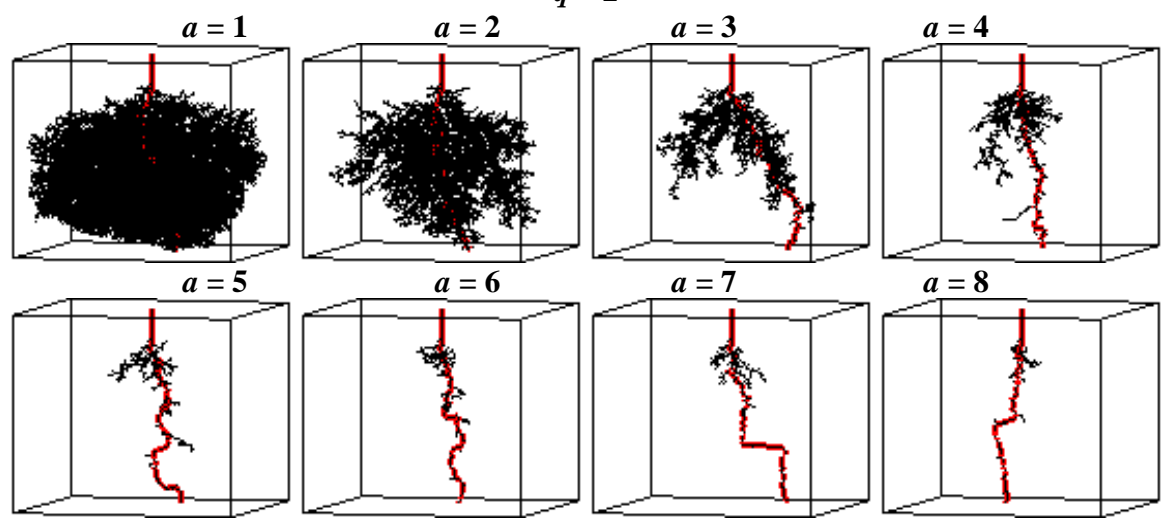

$q=3$
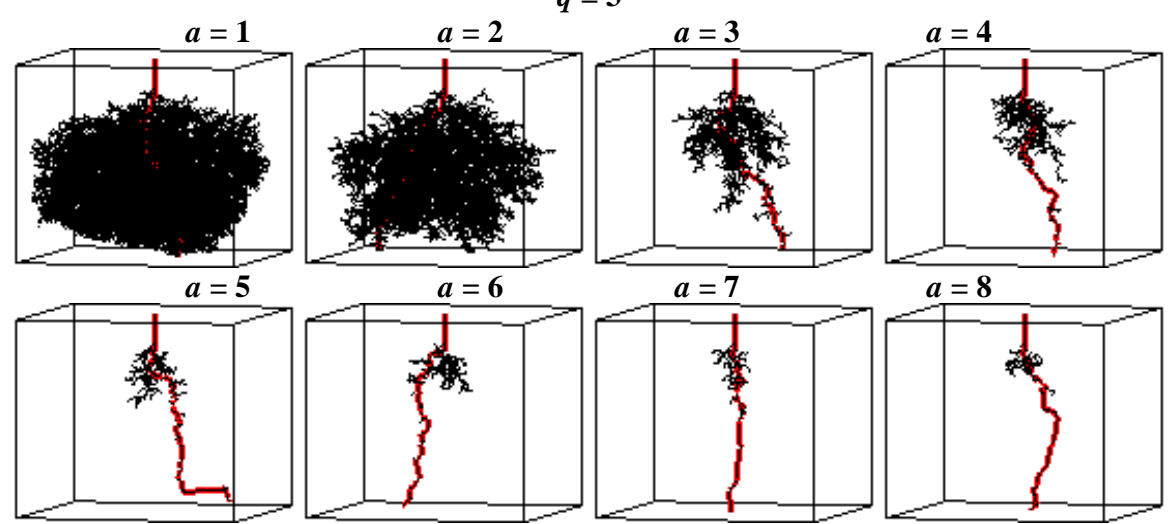

$a=8$

$q=4$
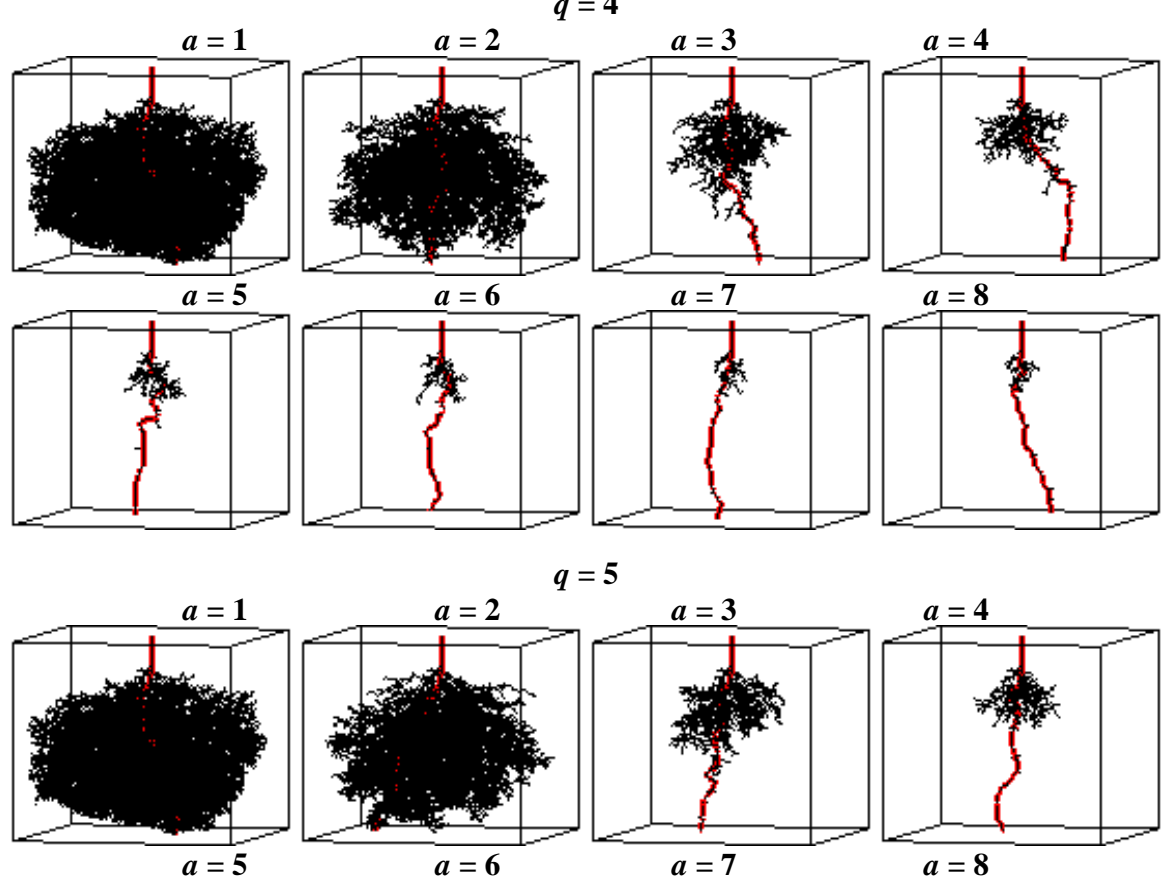

$q=5$

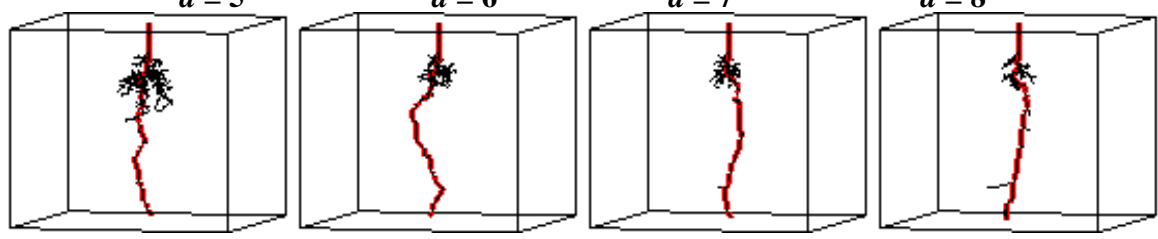

Figure 15. Example streamers generated with the two-parameter function for $p$ depicted in Figure 9. 
a)

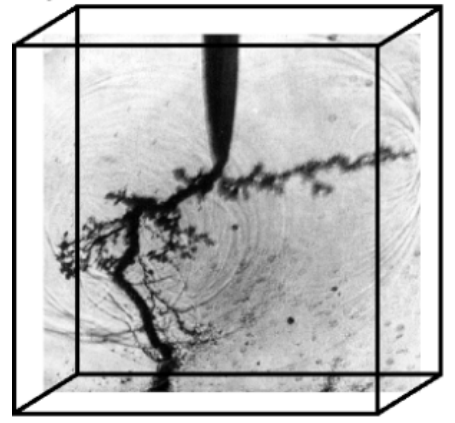

b)

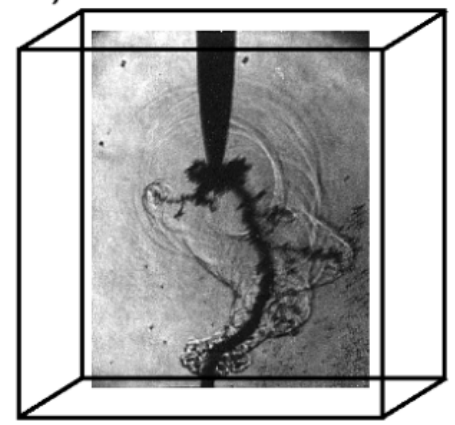

c)

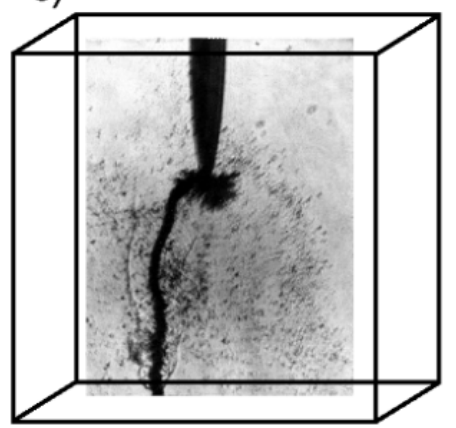

d)

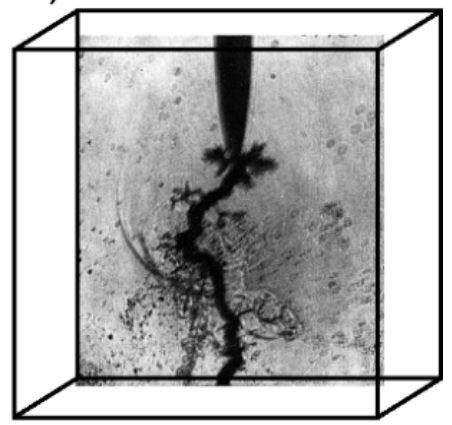

1501.0668

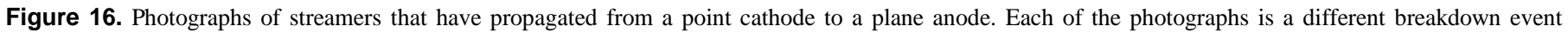

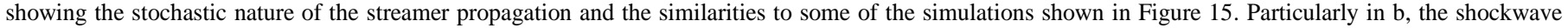

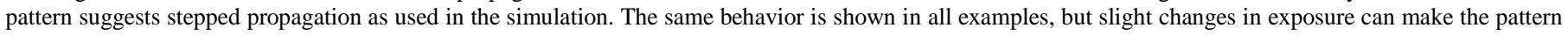
less clear in some of the examples.

The last remaining parameter to study is the power of exponentiation. Figure 13 shows that this parameter has a strong affect on the growth and appearance of the streamer. Exponentiation with a power greater than 1 will decrease the mean and increase the variance of the $\phi$ population. This increases the likelihood that growth points will be among the larger $\phi$ values in the population.

Figure 14 shows an example of a type of streamer that would be desirable to produce with the simulation model. Such a streamer exhibits a greatly accelerated growth rate in its final stages, producing a sparse streamer propagating toward the plane electrode. Figure 13 suggests a possible approach to achieving this in simulation is by having the exponential power be a function of distance across the gap. This was done using the dimensionless distance variable $L$ $=y / g$ for the length of the streamer (refer to equation (4) and Figure 8). Figure 9 shows a particular type using two parameters, found capable of producing streamers similar to that in Figure 14. Figure 15 shows a collection of example streamers generated with this model. This is the first demonstration, using this modeling approach, showing the transition from one streamer mode type to another, a phenomenon that is typical in experiments [6, 12, 13-16].

It is clear these simulations reproduce experimentally measured characteristics. A representative sample of measurements is shown in Figure 16. The figure shows streamers that initiate at a point cathode. They show the diversity of shapes that are found in the simulation results. The figure also suggests that physical growth, like the simulation, is accomplished through a series of steps. That is consistent with the multitude of discrete shock waves that are shown in the figure. The shock waves were not shown in the simulation as the physics necessary to produce them is not included in the model.

\section{CONCLUSIONS}

The primary new information from this investigation is the simulation of a transition from one streamer mode to another during propagation. This demonstrates that the conceptual framework initially developed in [1] is capable of simulating a wide range of streamer propagation behaviors in insulating liquids. The work also improved the approach in the model for scaling the potential at nearby grid points and explored some of the influences of computational choices, specifically needle length and grid size, on the predicted results.

The modeling refinement that leads to the observation of transition between modes was to recognize that, on the scale of the individual streamers that constituted the "bush" structure, that the shielding of the electric field by adjacent streamers was incomplete. Consequently, the various grid points had somewhat different potentials. A computational bias was established so that the streamers would grow preferentially in the direction of the largest potential differences and not strictly randomly. It was assumed that this bias would be consistent with physical processes. The assumed bias was sufficient to exhibit mode transition.

An important analysis that still needs to be done within the framework of this model is to use experimental data to infer the production rates of available electrons. With that data, it should be possible to replicate the measured mode propagation velocities. Moreover, adding this physics of the process should make the results much less sensitive to the selection of grid size.

Like any modeling approach, this approach is valuable to the extent that it provides a consistent way to provide insight into a range of data. So, while it is possible, or even likely, that this is not the best approach to describing the situation, this investigation helps to expand the range of phenomena that the model naturally describes.

\section{ACKNOWLEDGMENT}

This work was supported in part by the Office of Naval Research. The authors thank Dr. Minkyu Kim for valuable discussions on streamer simulation.

\section{REFERENCES}

[1] H. Fowler, J. Devaney, and J. Hagedorn, "Growth model for filamentary streamers in an ambient field", IEEE Trans. Dielectr. Electr. Insul., Vol. 10, pp. 73-79, 2003.

[2] M. Kim and R. E. Hebner, "Initiation from a point anode in a dielectric liquid”, IEEE Trans. Dielectr. Electr. Insul., Vol. 13, pp. 1254-1260, 2006. 
[3] M. Kim, R. E. Hebner, and G. A. Hallock, "Modeling the growth of streamers during liquid breakdown”, IEEE Trans. Dielectr. Electr. Insul., Vol. 15, p 547-553, 2008.

[4] E. F. Kelley and R. E. Hebner, "The electric field distribution associated with prebreakdown phenomena in nitrobenzene”, J. Appl. Phys., Vol. 52, pp. 191-195, 1981.

[5] W. F. Schmidt, M. Kim, and R. E. Hebner, "Initiation from a point anode in a dielectric", IEEE Trans. on Dielectr. Electr. Insul., Vol. 14, pp. 762-763, 2007.

[6] R. E. Hebner, "Measurement of electrical breakdown in liquids", Proc. of the NATO Advanced Study Institute on the Liquid State and its Electrical Properties, Sintra, Portugal, Plenum Pub. Corp., New York City, NY, pp. 519-537, 1987.

[7] H. Yamashita, K. Yamazawa, and Y. S. Wang, "The effect of tip curvature on the prebreakdown streamer structure in cyclohexane", IEEE Trans. Dielectr. Elect. Insul., Vol. 5, pp. 396-401, 1998.

[8] P. Gournay and O. Lesaint, "A study of the inception of positive streamers in cyclohexane and pentane”, J. Phys. D: Appl. Phys., Vol. 26, pp. 1966-1974, 1993.

[9] W. Gropp, E. Lusk, and A. Skjellum, Using MPI - Portable Parallel Programming with the Message-Passing Interface, The MIT Press, Cambridge, Massachusetts, 1994.

[10] E. F. Kelley and R. E. Hebner, "The electrical field distribution associated with prebreakdown phenomena in nitrobenzene”, J. Appl. Phys., Vol. 52, pp. 191-195, 1981.

[11] A. H. Sharbaugh, J. C. Devins, and S. J. Rzad, "Progress in the field of electrical breakdown in liquid dielectrics", IEEE Trans. Electr. Insul., Vol.13, pp. 249 -2276, 1978.

[12] M. Zahn, E. Forster, E. Kelley, and R. Hebner, "Hydrodynamic shockwave propagation after electrical breakdown", J. Electrostatics, Vol. 12, pp. 535-546, 1982.

[13] R. E. Hebner, E. F. Kelley, E. O. Forster, and G. J. FitzPatrick, "Observation of prebreakdown and breakdown phenomena in liquid Hydrocarbons II. Non-uniform field conditions”, IEEE Trans. Electr. Insul., Vol. 20, pp. 281-292, 1985.

[14] G. J. Fitzpatrick, E. O. Forster, R. E. Hebner, and E. F. Kelley, "Prebreakdown cathode processes in liquid hydrocarbons," IEEE Trans. on Electr. Insul., Vol. 22, pp. 453-458, 1987.

[15] P. J. McKenny, E. O. Forster, E. F. Kelley, and R. E. Hebner, "Effect of pressure on the development of prebreakdown streamers”, IEEE Conf. Electr. Insul. Dielectr. Phenomena, pp. 263268, 1988.

[16] E. F. Kelley and R. E. Hebner, "Prebreakdown phenomena between sphere-sphere electrodes in transformer oil”, Appl. Phys. Letters, Vol. 38, pp. 231-233, 1981.

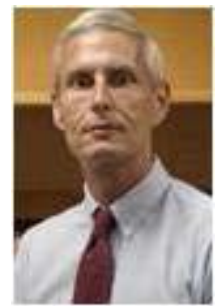

Brian T. Murphy earned his mechanical engineering degrees from the University of Florida in 1978, and Texas A\&M University in 1984. He worked for nine years in the aerospace industry at the Rocketdyne division of Rockwell International working on rocket engine turbomachinery, including the main engines of the Space Shuttle. Since 1993 he has been working as a research scientist at the Center for Electromechanics of the University of Texas in Austin, Texas. The Center performs fundamental and applied research in electromagnetics, electromechanical devices, power electronics, and advanced materials. This includes large scale high speed motors, generators and energy storage flywheels.

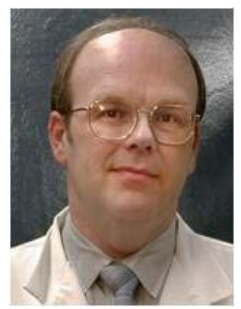

Edward F. Kelley graduated from the University of Idaho in 1970 with a degree in physics. He received the Ph.D. degree in experimental atomic physics from Montana State University in 1977. He started in a post-doctoral position at NIST in high-voltage impulse measurements using the electro-optical Kerr effect. He continued at NIST as a staff member for approximately 11 years, investigating liquid dielectric breakdown and highvoltage pulse-measurement techniques. In 1988, he received the R\&D 100 award for an Image Preserving Optical Delay designed for photographing random events before they happen but after they occur. After going to Idaho to get a taste of private consultation and university teaching, he returned to NIST in 1992 to work in the Flat Panel Display Laboratory of the Display Metrology Project to assist industry in developing measurement standards to quantify display quality. He has served as chair and main author of the Display Metrology Committee of VESA that produced the Flat Panel Measurements Standard (FPDM) and continues as editor and main author of its extension, the Display Measurements Standard, under the International Committee for Display Metrology of the Society for Information Display.

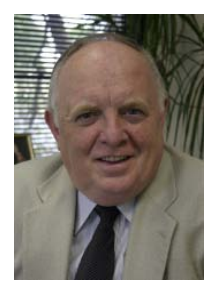

Robert E. Hebner, Ph.D., (S'70-M'71-SM'83-F'93) is Director of the Center for Electromechanics at the University of Texas at Austin. The Center develops technology, primarily novel motors, generators, and suspension components, and teams with companies to get the technology into the market. Previously, Dr. Hebner was the acting Director of the U.S. National Institute of Standards and Technology (NIST). In addition, he has directed NIST's Electronic and Electrical Engineering Laboratory, a laboratory with a staff of more than 250. He also worked at the Defense Advanced Research Projects Agency where he developed programs to improve semiconductor manufacturing. Throughout his career, Dr. Hebner has been active in having authored or coauthored more than one hundred technical papers and reports. He has extensive experience in international technology programs. This work included the modernization of the measurement systems needed to support global trade and the assessment of the effectiveness of government technology programs in stimulating domestic economies. 